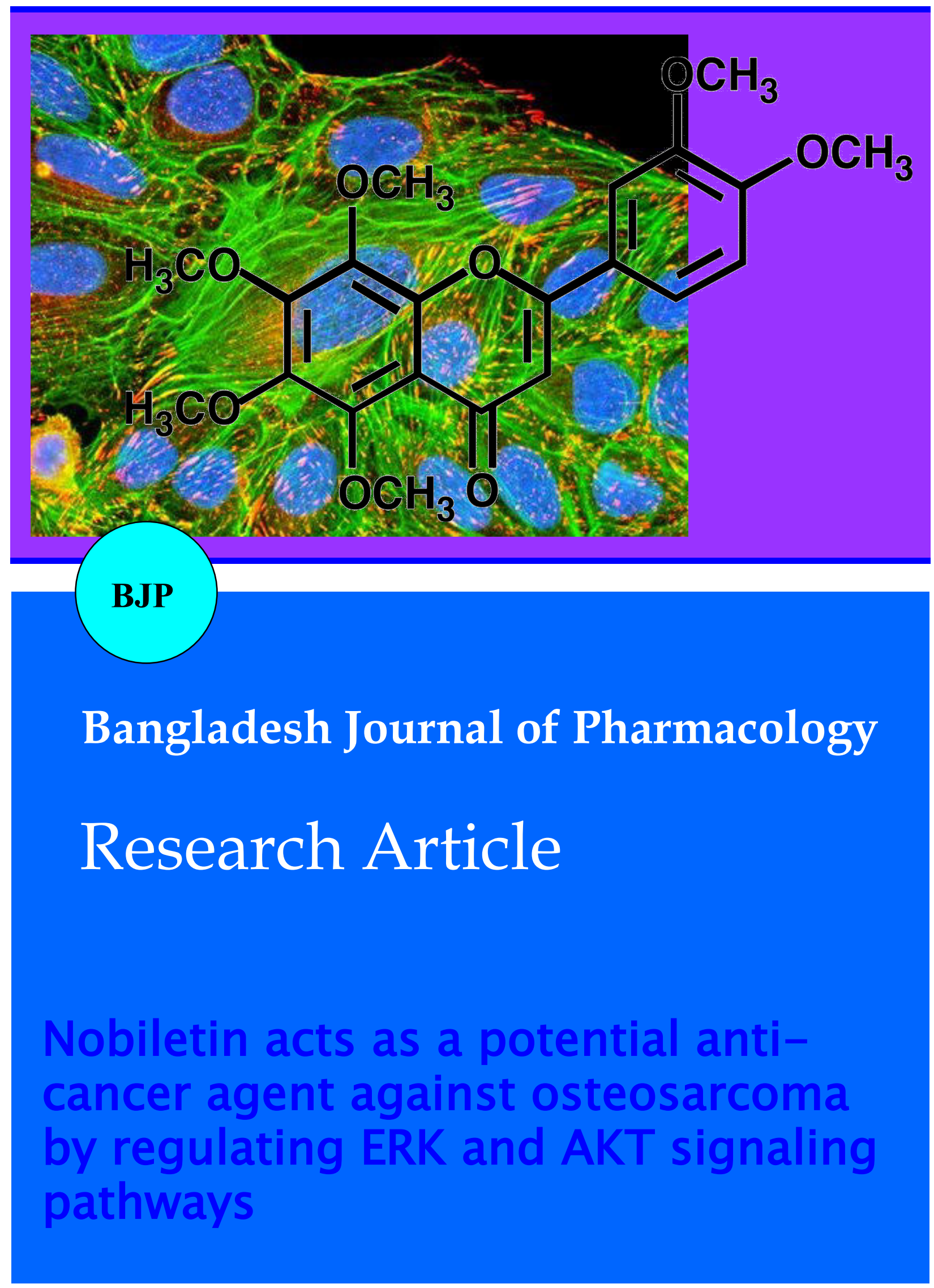




\title{
Nobiletin acts as a potential anti-cancer agent against osteosarcoma by regulating ERK and AKT signaling pathways
}

\author{
Fu Wen Niu', Yong Jie Zhang', Kun Li3,4 and Mao Shu Zhang1 \\ ${ }^{1}$ Department of Orthopedics, Jining No.1 People's Hospital in Shandong Province, P.R. China; ${ }^{2}$ Department of \\ Orthopedics, JinXiang People's Hospital in Shandong Province, P.R. China; ${ }^{3}$ Department of Gynecology and \\ Obstetrics, Affiliated Hospital of Shandong Academy of Medical Sciences, P.R. China; ${ }^{4}$ Department of Gynecology \\ and Obstetrics, Shandong Academy of Medical Sciences, P.R. China.
}

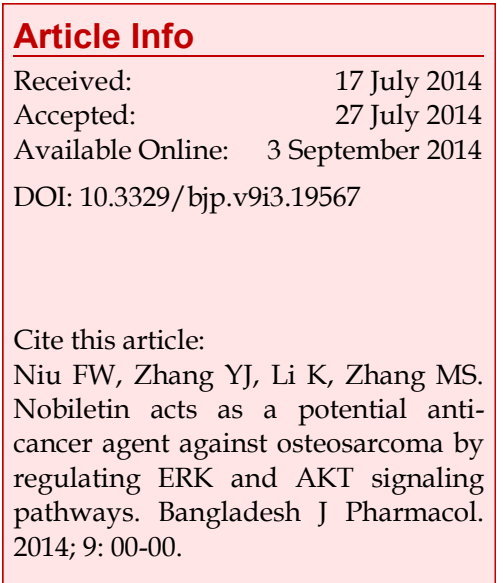

\begin{abstract}
The current study was aimed to evaluate the anti-proliferative activity of nobiletin, a flavonoid, on osteosarcoma cell line, SaOS-2. Furthermore, we attempted to investigate the signaling pathways involved in the cell growth inhibition mechanism. Upon exposure, nobiletin showed significant cell inhibitory effect on SaOS-2 cells in a dose-dependent manner as evidenced by MTT assay and LDH assay. Moreover, flow cytometric analysis demonstrated that upon treatment with nobiletin, SaOS-2 cells underwent apoptosis and showed increased number of cells arrested in G2/M phase. Activation of caspase- 9 and -3 indicate the activation of apoptotic signaling pathway in SaOS-2 cells. Additionally, it was observed that the cell inhibitory effect was regulated by the activation of ERK signaling pathway while AKT signaling pathway was down regulated in SaOS-2 cells after 24 hours of Nobiletin treatment.
\end{abstract}

\section{Introduction}

Skeletal integrity is maintained by equilibrium between osteoblasts mediated bone formation and osteoclasts mediated bone resorption. Any imbalance in this equilibrium leads to osteoblastic lesions and other severe clinical consequences often termed as bone cancer or osteosarcoma. Commonly, bone cancer is resulted by invasion of different cancers to bone tissue, phenomenon known as bone metastases. American cancer society estimates nearly 3,020 new cases of bone cancer and 1,460 deaths due to bone cancer in 2014 (American Cancer Society, 2014). Although, many chemotherapeutic drugs are currently employed for the treatment of bone cancer, yet there is demand for safe and novel drugs having minimum side effects. Over the past few years, the development of natural compounds as anticancer agents has been revealed (Newman and Cragg, 2007). Intensive efforts for discovering new drugs based on natural products has proven beneficial, with nearly
$47 \%$ drugs of natural origin been approved as anticancer agents. Flavonoids are a group of natural polyphenols widely present in plants and well known to play significant role in various pharmacological activities (Harborne and Williams, 2000; Kim et al., 2013). Recent studies highlighted the anti-cancer potential of these flavonoids and explored as therapeutic drugs in clinics (Neuhauser, 2004).

Nobiletin (5,6,7,8,3',4'-hexamethoxy flavone), a flavonoid, is present in citrus fruits and found to possess anti -inflammatory (Murakami et al., 2005) and antitumor potential (Tang et al., 2007; Sato et al., 2002). Previous studies showed the anti-proliferative effect of nobiletin on various cell lines including lung adenocarcinoma cell line A549 cells (Luo et al., 2008) and hepatic cancer cell line, SMMC-7721 (Ma et al., 2014). However, the anti-proliferative effect of nobiletin on osteosarcoma cells has not been studied till date. Furthermore, the mechanism of anti-cancer action exerted by nobiletin is 
also rarely reported. Therefore, this study aims to characterize the inhibitory effect of nobiletin on osteosarcoma cell line and delineate the signaling mechanism involved.

\section{Materials and Methods}

\section{Materials}

Nobiletin (purity $\geq 98 \%$ ), 3-(4,5-dimethlthiazol-2-yl)-2,5diphenyl-tetrazoliumbromide (MTT), bovine serum albumin (BSA), fetal bovine serum (FBS), phosphate buffer saline (PBS), DMSO, sodium dodecyl sulfate (SDS), EDTA, Tris- $\mathrm{HCl}$, was purchased from SigmaAldrich (USA). Cytotoxicity detection kit was purchased from Takara Bio Inc., Shiga, Japan. Caspase-3 colorimetric assay kit and caspase- 9 colorimetric assay kit was obtained from Biovision, Inc. Protein assay reagent was purchased from Bio-Rad Laboratories (Hercules, USA). Antibody against AKT, ERK, $\beta$ catenin and $\beta$-actin were purchased from Cell Signaling Technology, Danvers, MA.

\section{Cell culture}

Human osteosarcoma cell line, SaOS-2, was cultured in $10 \%$ fetal bovine serum (FBS), $100 \mathrm{U} / \mathrm{mL}$ penicillin and $100 \mu \mathrm{g} / \mathrm{mL}$ streptomycin (Lonza, Switzerland) supplemented a-MEM medium (Invitrogen, USA). The cells were grown at $37^{\circ} \mathrm{C}$ in humidified chamber with $95 \%$ air and $5 \% \mathrm{CO}_{2}$. The confluence level of SaOS-2 cell line was maintained at $70-80 \%$. SaOS-2 cell line was chosen because it is known to be depicting osteosarcoma characteristics and is being widely used for cell proliferation and differentiation studies (Hunt et al., 1996).

\section{Cell viability assay (MTT assay)}

The anti-proliferative effect of nobiletin on SaOS-2 cell line was performed by MTT assay. Cell viability assay was achieved by plating $2 \times 10^{4}$ cells/well in 96 well plate at $37^{\circ} \mathrm{C}$ for 24 hours followed by treatment with different doses of nobiletin $(0.5,1,2.5,5$ and $10 \mu \mathrm{M})$. After incubating the cells at predetermined time points i.e. 24, 48 and 72 hours, 3-(4,5-dimethlthiazol-2-yl)-2,5diphenyl-tetrazoliumbromide (MTT) solution $(20 \mu \mathrm{L})$ was added in each well and again incubated for 3 hours at $37^{\circ} \mathrm{C}$ to facilitate the production of MTT formazan by mitochondrial succinate. After removal of supernatant, DMSO was added to each well to dissolve MTT formazan. Cell viability was calculated after measuring optical density using UV-Vis spectrophotometer at $570 \mathrm{~nm}$ wave length. Each experiment set was done in triplicate for accuracy and data are expressed as mean \pm SD.

\section{LDH assay}

Cell cytotoxicity was determined by evaluating the release of lactate dehydrogenase enzyme from the damaged cells using cytotoxicity detection kit according to manufacturer's protocol. Lactate dehydrogenase enzyme is found in the cytoplasm of cells and is commonly used as a marker to detect cell membrane damage (Legrand et al., 1992). Briefly, SaOS-2 cells were seeded in 96 wells plate at cell density of $2 \times 10^{4}$ cells/ well and further incubated at $37^{\circ} \mathrm{C}$. After 24 hours, cells were treated with various doses $(0.5,1,2.5,5$ and 10 $\mu \mathrm{M})$ of nobiletin for $0,24,48$ and 72 hours. From each well, cell culture medium was collected and mixed with PBS and LDH reagent in ratio of 1:4:5 and kept in dark for 1 hour at $25^{\circ} \mathrm{C}$. Following the addition of $50 \mu \mathrm{L}$ of stop solution, absorbance was measured at $490 \mathrm{~nm}$.

\section{Flow cytometric study for apoptosis evaluation}

After SaOS-2 cells were treated with nobiletin at $0.5,1$, $2.5,5$ and $10 \mu \mathrm{M}$ doses for 24 hours, cells were harvested and collected as pellet (1,000 rpm for $5 \mathrm{~min})$. Subsequently, cells were fixed with $70 \%$ ice cold ethanol and washed twice with PBS. Cells were further treated with Triton X-100 $(1 \% \mathrm{v} / \mathrm{v})$ and incubated at $37^{\circ}$ $\mathrm{C}$ for $10 \mathrm{~min} .50 \mu \mathrm{g} / \mathrm{mL}$ of propidium iodide solution was then added to the cells at $4^{\circ} \mathrm{C}$ for $15 \mathrm{~min}$ in the darkto stain the cellular DNA. The distribution of cells in the phases of cell cycle and apoptotic rate was then determined using FACScan flow cytometer (BD Biosciences, San Jose, CA, USA)

\section{Western blot analysis}

Total cell lysate was collected fromSaOS-2 cells treated with nobiletin for 24 hours in ice cold RIPA buffer containing protease inhibitor cock-tail (Roche). Thereafter, samples were vortexed and centrifuged at 12,000 rpm for $30 \mathrm{~min}$ to collect the supernatant. Total protein amount was determined using protein assay reagent (Bio-Rad) to normalize the protein content. Equal amount of proteins were separated on $10-12 \%$ sodium deodecyl sulfate-polyacrylamide gel electrophoresis and then electrophoretically transferred to nitrocellulose membrane. Tween 20-tris buffered saline (TBST) including nonfat dry milk (5\%) was spread on the membrane at room temperature for 1 hour to block non-specific sites and then the membrane was incubated overnight with primary antibodies (pAKT, pERK, ERK, AKT and $\beta$-actin) at $4^{\circ} \mathrm{C}$. The membrane was washed again with TBST three times and then incubated with appropriate horseradish peroxidase (HRP) conjugated rabbit anti-mouse secondary antibody (Santa Cruz Biotech, CA, USA) for 1 hour at room temperature. Again after three times washing the membrane with TBST, bands were visualized by enhances chemiluminescence detection system (FUJFILM Las3000 mini, Tokyo, Japan).

\section{Caspase-3 and -9 activity assay}

To confirm the apoptotic activity, caspase- 3 and -9 were determined using caspase- 3 and -9 colorimetric assay 
kit, according to manufacturer's protocol. After the treat -ment of SaOS-2 cells with nobiletin, total cells were collected as pellet by centrifuging at $12,000 \times \mathrm{g}$ for 10 min. Pellet was then resuspended in $50 \mu \mathrm{L}$ of ice cold lysis buffer for $10 \mathrm{~min}$ at $4^{\circ} \mathrm{C}$ and centrifuged again at $10,000 \times \mathrm{g}$ for $1 \mathrm{~min}$ to collect supernatant. After the quantification of total protein in the supernatant, respective substrates were added to equal amount of proteins and incubated at $37^{\circ} \mathrm{C}$ for $30 \mathrm{~min}$. Activity was determined by reading the samples at $400 \mathrm{~nm}$ in a microtiter plate reader.

\section{Over expression of AKT allele}

SaOS-2 Cells were plated at $3.5 \times 10^{4}$ cells/well in a 48 well dish and transfected with $0.75 \mu \mathrm{g}$ of myrAktdeltaPH plasmid DNA (Addgene, Cambridge, MA:10841), encoding Myr-Akt, or empty plasmid pECE (Addgene:26453), as control, with Fugene HD (Promega, Sunnyvale, CA)transfection reagent according to the manufacturer's protocol. After 8 hours of transfection, cells were treated with nobiletin for 24 hours. MTT assay was performed to assess the cell viability of treated cells as described above.

\section{Inhibition of ERK signaling pathway}

SaOS-2 Cells plated at a density of $3.5 \times 10^{4}$ cells/well in a 48-well dish were treated with nobiletin, with or without PD98059 $(5 \mu \mathrm{M})$ for 24 hours. Cell viability of SaOS-2 cells was analyzed by MTT assay.

\section{Statistical analysis}

GraphPad Prism 5.0 (San Diego, CA) was utilized for evaluating statistical analysis of obtained data by using two-tailed Student t-test. Statistical significance was considered at a value of $\mathrm{p}<0.05$.

\section{Results}

The anti-proliferative effect of nobiletin on osteosarcoma cell line was determined following 24, 48 and 72 hours treatment of SaOS-2 cells with nobiletin at vari- ous doses of $0.5,1.0,2.5,5.0$ and $10.0 \mu \mathrm{M}$ using MTT assay. Results demonstrated growth inhibitory effect of nobiletin on SaOS-2 cells in a dose-dependent manner at all the time points (Figure 1A). After 24 hours of treatment, SaOS-2 cell viability was decreased to 50 and $70 \%$ of control by nobiletin at a dose of 5 and $10 \mu \mathrm{M}$, respectively. Even low doses of nobiletin $(0.5,1$ and 2.5 $\mu \mathrm{M})$ showed significant growth inhibitory effect on SaOS-2 cells compared to control at 72 hours. In agreement to MTT assay, dose-dependent cell cytotoxicity exerted by nobiletin was also demonstrated by LDH assay (Figure 1B).

To examine whether nobiletin treatment could affect cellcycle progression and apoptosis in osteosarcoma cells, SaOS-2 cells were treated with different concentrations of nobiletin. Rate of apoptosis and phase of cell cycle arrest induced by nobiletin on SaOS-2 cells was analyzed using flow cytometry after 24 hours of treatment. As shown in Table I, $28.2 \pm 2.2 \%$ increase in rate of apoptosis was observed after treatment with $10 \mu \mathrm{M}$ of nobiletin (Figure 2A). In addition, cells treated with lower doses of nobiletin also showed increased rate of apoptosis in a dose-dependent manner. An increase in the number of cells arrested at G2/M phase in SaOS-2 cells was observed after treatment of nobiletin in a dose -dependent manner (Figure 2B).

Following 24 hours treatment of SaOS-2 cells with different doses of nobiletin, caspase- 9 and -3 activities were determined. Results demonstrated a significant increase of caspase- 9 and -3 activities after stimulation with nobiletin (Figure 3). A dose-dependent increase in activities was observed for both, caspase- 9 and -3 , after 24 hours of stimulation with nobiletin in SaOS-2 cells (Figure 3A and B). An increment in the caspase-9 and 3 activity points toward a caspase- 9 and -3 dependent signaling in SaOS-2 cells for inducing apoptosis by nobiletin.

Results from Western blotting showed (Figure 4) that the protein levels of p-ERK were upregulated in SaOS-2 cells after treatment with nobiletin, but that of $\mathrm{p}-\mathrm{AKT}$

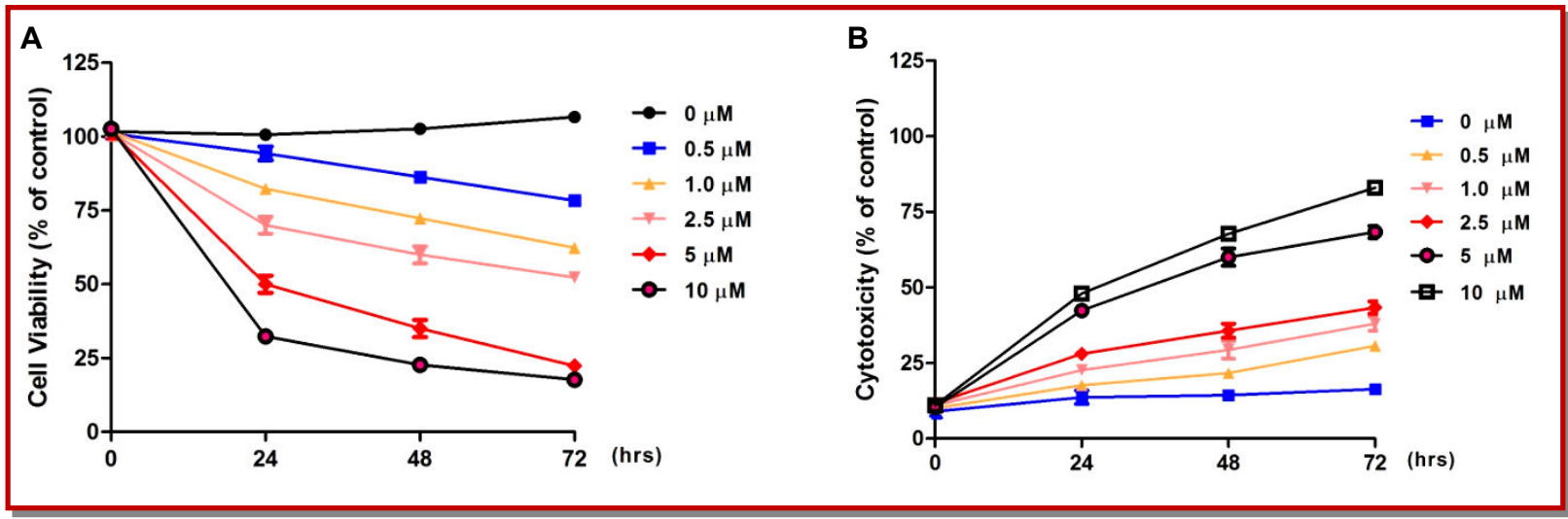

Figure 1: Anti-proliferative effect of nobiletin at different concentrations on SaOS-2 cells (A) MTT assay (B) LDH assay 


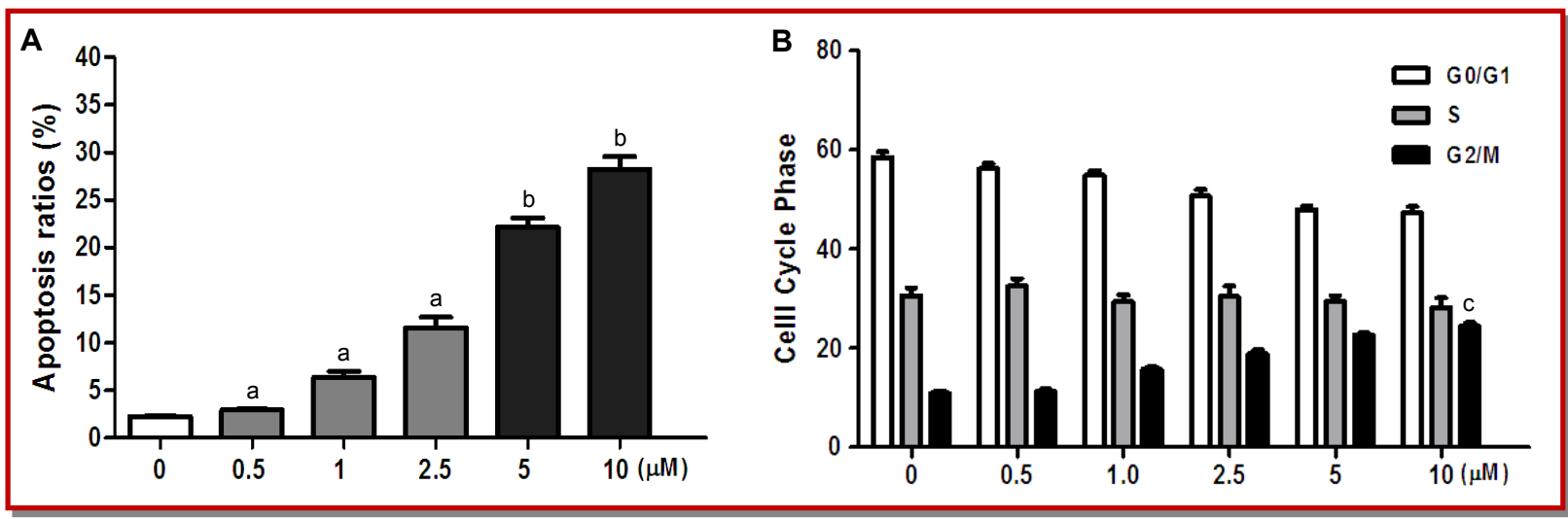

Figure 2: Effect of nobiletin treated for 24 hours at different concentration $(0,0.5,1,2.5,5$ and $10 \mu \mathrm{M})$ on apoptosis and cell cycle arrest. (A) Nobiletin induces apoptosis ratio in SaOS-2 cells; (B) Cell cycle distribution after treatment of SaOS-2 cells with varying doses of nobiletin. Three independent experiments were performed and data is expressed as their mean $\pm \operatorname{SD}\left({ }^{\mathrm{a}} \mathrm{p}<0.01\right.$, $\left.{ }^{\mathrm{b}} \mathrm{p}<0.001\right)$

\begin{tabular}{|c|c|c|c|c|c|}
\hline \multicolumn{6}{|c|}{ Table I } \\
\hline \multicolumn{6}{|c|}{ The cell cycle and apoptosis ratios with various concentrations of nobiletin on SaSO-2 cells } \\
\hline Group & Concentration $(\mu \mathrm{M})$ & $\mathrm{G}_{\mathrm{o}} / \mathrm{G}_{1}(\%)$ & $\mathrm{S}(\%)$ & $\mathrm{G}_{2} / \mathrm{m}(\%)$ & Apoptosis ratio (\%) \\
\hline \multirow{6}{*}{ Nobiletin } & 0 & $58.5 \pm 1.5$ & $30.5 \pm 2.7$ & $10.9 \pm 0.7$ & $2.2 \pm 0.0$ \\
\hline & 0.5 & $56.2 \pm 1.4$ & $32.5 \pm 2.6$ & $11.2 \pm 0.8$ & $2.9 \pm 0.1$ \\
\hline & 1.0 & $54.9 \pm 1.4$ & $29.3 \pm 2.3$ & $15.7 \pm 0.8$ & $6.3 \pm 0.0$ \\
\hline & 2.5 & $50.6 \pm 2.0$ & $30.4 \pm 3.6$ & $18.8 \pm 1.3$ & $11.5 \pm 1.9$ \\
\hline & 5.0 & $47.9 \pm 1.3$ & $27.4 \pm 1.9$ & $22.6 \pm 0.7$ & $22.1 \pm 1.6$ \\
\hline & 10 & $47.3 \pm 2.1$ & $28.2 \pm 3.2$ & $24.4 \pm 1.2$ & $28.2 \pm 2.2$ \\
\hline
\end{tabular}
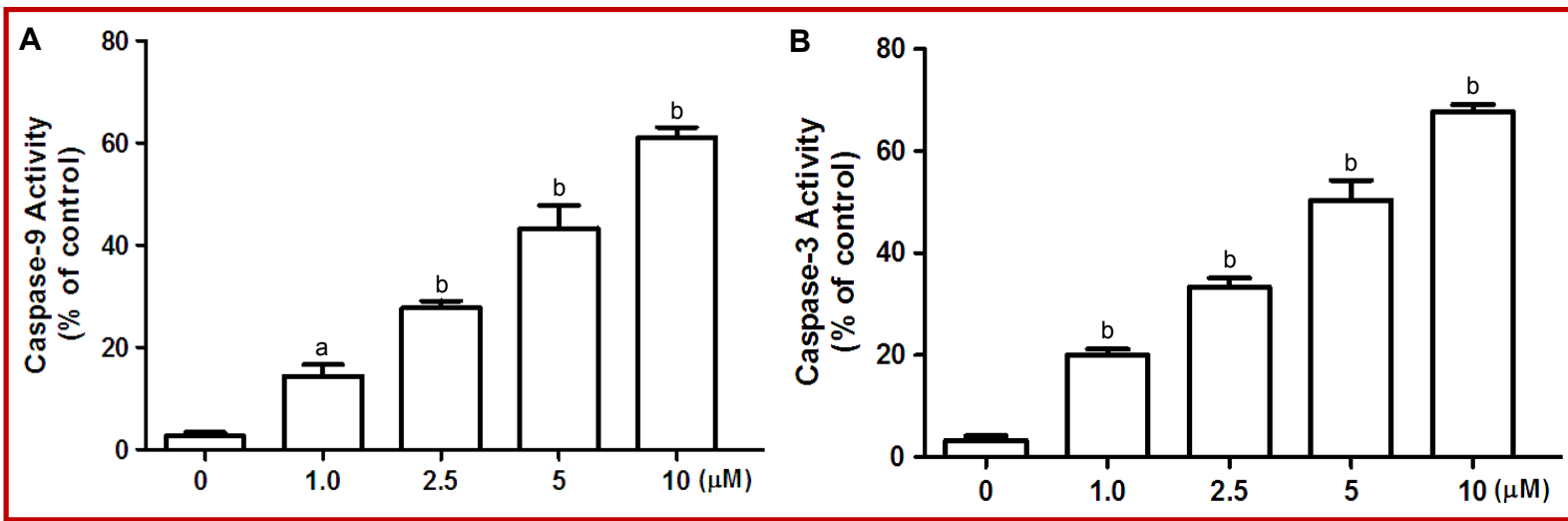

Figure 3: Activation of caspase activity after 24 hours of treatment with varying doses of nobiletin, (A) Caspase-9 and (B) Caspase3 activity. Three independent experiments were performed and data is expressed as their mean $\pm \operatorname{SD}\left({ }^{a} p<0.01,{ }^{b} p<0.001\right)$

was down-regulated. Phosphorylation of ERK molecules were in a dose-dependent manner after 24 hours of treatment. Similarly, a dose-dependent suppression of phosphorylation of AKT molecule was observed after 24 hours of treatment.

In order to substantiate our results that nobiletin mediates it anti-proliferative effect through activation of ERK signaling pathway and suppression of AKT signaling, we tried to inhibit the activation of ERK pathway by PD 98059 and restore AKT signaling in SaOS-2 by constitutive expression of AKT molecule in presence of nobiletin. ERK signaling pathways was inhibited by well-known MEK inhibitor, PD98059 (5 $\mu \mathrm{M})$, for $30 \mathrm{~min}$ prior to the treatment of nobiletin (5 $\mu \mathrm{M})$ to SaOS-2 cells. After 24 hours of treatment, MTT assay was performed to assess the cell viability of the treated cells. As shown in Figure 5A, nobiletin $(5 \mu \mathrm{M})$ was able to induce $50 \%$ decrease in cell viability compared to control whereas prior blocking of ERK signaling by PD98059 dramatically reversed the cytotoxicity induced by nobiletin in comparison to only nobiletin treated SaOS-2 cells. To deduce the role AKT signaling pathway, SaOS-2 cells were transiently 


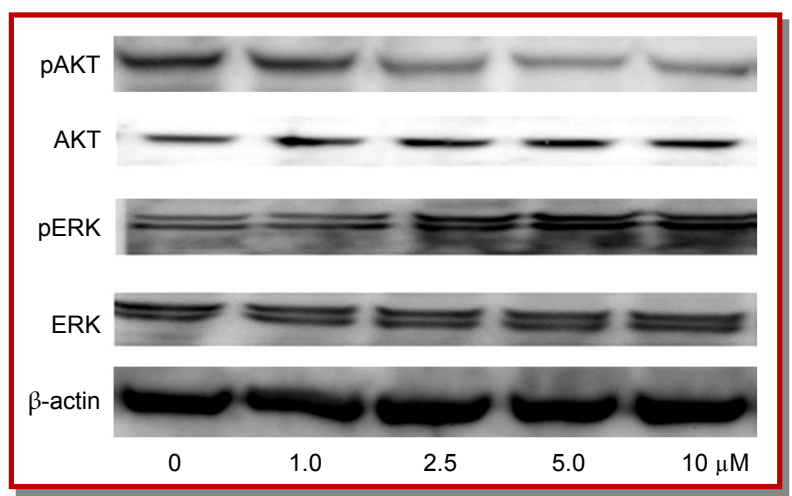

Figure 4: Nobiletin regulated expression of pAKT, AKT, pERK, ERK and $\beta$-actin in SaOS-2 cells

transfected with myrAktdeltaPH plasmid DNA followed by treatment of nobiletin for 24 hours. Expression of constitutive form of AKT inside the SaOS-2 cells nullified the anti-proliferative effect of nobiletin (Figure $5 \mathrm{~B})$, restoring proliferative character of SaOS-2 cells.

\section{Discussion}

Dietary phytochemicals has been shown to possess various beneficial biological properties including anticancerous. For instance, intake of citrus fruits has been considered not only beneficial for health but even possess anti-carcinogenic and anti-tumor activities (Gullett et al., 2010; Wu et al., 2013). Among numerous active flavonoids in citrus fruits, nobiletin has been reported to possess potent biological activities. Previous studies have described various biological effects of nobiletin like anti-inflammatory, anti-tumor, anti-metas -tasis and neuroprotective properties (Murakami et al., 2000; Nakajima et al., 2007; Li et al., 2006; Shi et al., 2013). Recent studies have also demonstrated compelling role of nobiletin in initiating anti-cancerous proper -ties in various cell culture systems (Luo et al., 2009; Ishii et al., 2010). In order to observe any effect of nobi- letin on bone cancer cells, MTT and LDH assay was performed (Figure 2). Results demonstrated dose-depen -dent inhibitory effect of nobiletin on SaOS-2 after 24, 48 and 72 hours treatment of SaOS-2 cells with nobiletin at various doses of $0.5,1.0,2.5,5.0$ and $10.0 \mu \mathrm{M}$.

Tumor cells proliferation depends on completed cell cycles (Hartwell and Kastan, 1994), regulated and controlled by G1/S and G2/M checkpoints. These checkpoints are responsible for triggering mitotic spindle assembly formation and ensure that other downstream activities are commenced only when upstream activities are correctly completed (Hartwell and Weinert, 1989). Regulation of cell cycle checkpoint also eliminates impaired cells either by inducing apoptosis or blocking cell cycle progression (causing apoptosis). Nobiletin has been demonstrated to possess anti-proliferative properties by promoting apoptosis and cell cycle arrest (Luo et al., 2008, Luo et al., 2009; Ishii et al., 2010). Thus, to confirm the effect of nobiletin on apoptosis and cell proliferation of SaOS-2 cells flow cytometry was performed. The results showed increased rate of apoptosis in a dose-dependent manner with increase in cell number arrested in G2/M phase of cell cycle. Taken together, results demonstrated that nobiletin induces anti-proliferative effect on osteosarcoma cells, SaOS-2, by inducing cell cycle arrest and apoptosis, similar to reported effect in hepatocellular carcinoma cells (Ma et al., 2014).

Two major signaling pathways that are involved in apoptotic cell deathare intrinsic pathway (mitochondria pathway) or extrinsic pathways (death receptor pathway) (Kroemer et al., 2007; Lai et al., 2007). The intrinsic pathway directs death signals to mitochondria by releasing mitochondrial inter-membrane proteins such as cytochrome c. Released cytochrome c in turn associates with apoptotic protease-activating factor-1 (Apaf-1) and pro-caspase- 9 to form the apoptosome, leading to the activation of caspase-3. On the other hand, extrinsic pathway acts through the activation of the cell surface
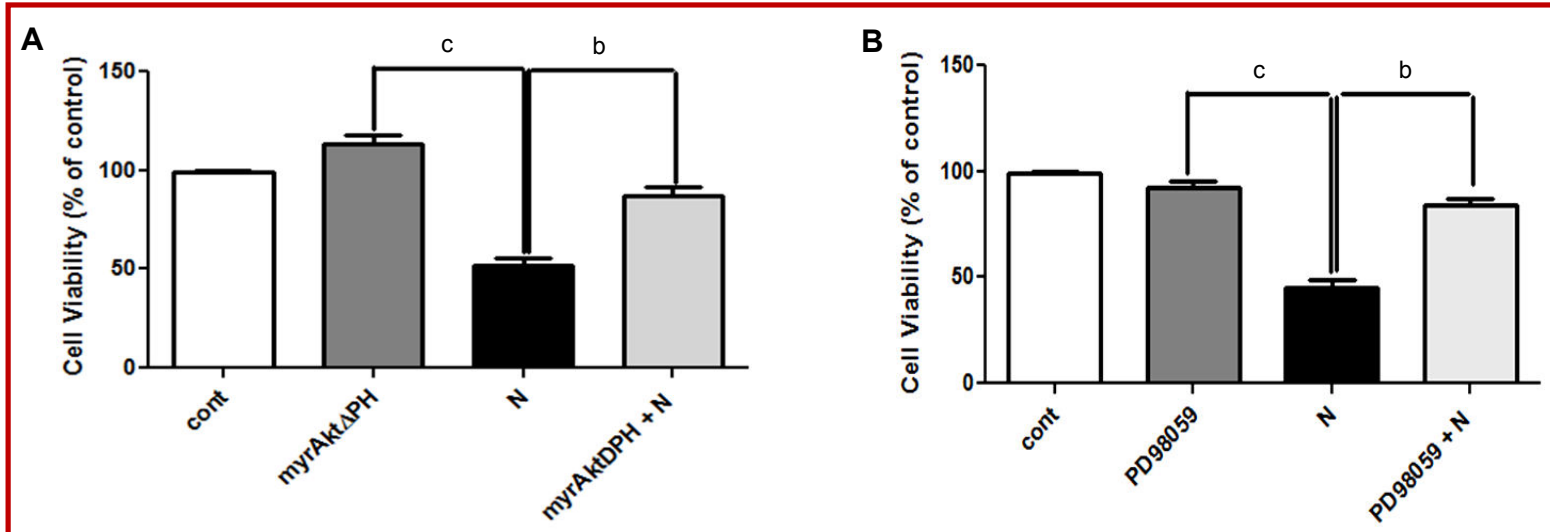

Figure 5: Anti-proliferative effect of nobiletin $(5 \mu \mathrm{M})$ on SaOS-2 cells after 24 hours of treatment (A) After blocking ERK signaling pathways by PD98059 (B) After transfecting SaOS-2 cells by myrAktdeltaPH plasmid DNA. Three independent experiments were performed and data is expressed as their mean $\pm \mathrm{SD}\left(\mathrm{a} p<0.05,{ }^{\mathrm{b}} \mathrm{p}<0.01, \mathrm{c} p<0.001\right)$ 
receptors through Fas/Fas ligand (FasL) or TNF-related apoptosis-inducing ligand (TRAIL) to promote caspase8 activation. Another signaling pathway independent of caspase pathway also exists which leads to cell death by releasing apoptosis inducing factor (AIF) or endonuclease G (Endo G) from mitochondria (Park, 2012). Thus, to determine whether nobiletin involves any of these pathways in inducing apoptosis in SaOS-2 cells, we first examined the possibility of activation of intrinsic pathway by measuring the activity of caspase- 9 and -3 in SaOS-2 cells. Results showed increase in caspase-9 and -3 activity indicating the role of intrinsic pathways in inducing apoptosis in SaOS-2 cells.

It is well documented that the activation of mitogenactivated protein (MAP)/extracellular signal-regulated kinase (MEK) is associated with anti-tumor activities. In addition, studies have shown that ERK/MAPK can positively mediate phosphorylation of Bcl-2 at Ser-70, causing anti-apoptotic function to down-regulate Bcl-2 expression. Activation of phosphatidyl inositol 3-kinase (PI3K) leads to phosphorylation of phosphatidyl inositides, which in turn activates the down-stream main target, $\mathrm{AKT}$, known to play various imperative roles in regulating cellular growth, adhesion, differentiation and the inflammatory reaction. Nobiletin has been reported to mediate inhibition of tumor promotion through regulation of MAPK/ERK and AKT protein kinase cascades (Shi et al., 2013, Aoki et al., 2013; Chen et al., 2014; Lai et al., 2008). Consequently, to elucidate whether nobiletin influences intrinsic apoptotic signaling through ERK or AKT signaling pathway, we performed western blot technique to check the phosphorylation status of ERK and AKT molecules after nobiletin treatment. The expression level of p-ERK, p-AKT, ERK and AKT suggests that the activation of ERK signaling pathway might be involved in stimulation of intrinsic pathway mediated by caspase- 9 and -3 , leading to apoptosis. Moreover, as AKT signaling pathways is associated with survival ability of any cells, a supperssion of phosphorylation of AKT signaling by nobiletin may be responsible for increased apoptosis observed in case of SaOS- 2 cells. These results were also examined and confirmed by inhibiting and restoring ERK and AKT signaling pathways, respectively.

Taken together, our results demonstrate that nobiletin can induce anti-proliferative effect on osteosarcoma cells by induction of cell cycle arrest and apoptosis by regulating ERK and AKT signaling pathways. This study results are in agreement with other researches which have successfully shown the anti-carcinogenic properties of nobiletin. Current finding clearly implicates nobiletin as a novel therapeutic agent for the treatment of bone cancers and further in vivo studies could establish it as one of the future drug for bone cancer treatment.
Chemoprevention constitutes an active cancer preventive strategy which could inhibit, delay or reverse human carcinogenesis. Numerous studies have provided substantial evidence that naturally products or synthetic chemical agents can block or reverse lethal changes in cellular signaling. Given the cytotoxic effects of synthetic chemical agents, natural agents are the need of hour for treating cancers. In line to this requirement, we have demonstrated that nobiletin can exert its anti-proliferative effect on osteosarcoma cells by regulating intrinsic pathway of apoptosis through regulation of ERK signaling pathway. Moreover, suppression of AKT signaling pathways further directs the cells toward cell death by nobiletin. Therefore, after in vivo and clinical trial, nobiletin can be regarded as future anti-cancerous agent to treat bone cancers.

\section{Acknowledgement}

This work was supported by Natural Science Foundation of Shandong Province: ZR2009CL027; Science and Technology Bureau of Jinan, Science and technology star fund project: 20100118; Shandong Academy of Medical Sciences Medical Foundation: 201023

\section{References}

American Cancer Society. Cancer: Facts and figures. Atlants, Ga, American Cancer Society, 2014.

Aoki K, Yokosuka A, Mimaki Y, Fukunaga K, Yamakuni T. Nobiletin induces inhibitions of Ras activity and mitogenactivated protein kinase kinase/extracellular signalregulated kinase signaling to suppress cell proliferation in C6 rat glioma cells. Biol Pharm Bull. 2013; 36: 540-47.

Chen C, Ono M, Takeshima M, Nakano S. Anti-proliferative and apoptosis-inducing activity of nobiletin against three subtypes of human breast cancer cell lines. Anti-cancer Res. 2014; 34: 1785-92.

Gullett NP, Ruhul Amin AR, Bayraktar S, Pezzuto JM, Shin DM, Khuri FR, Aggarwal BB, Surh YJ, Kucuk O. Cancer prevention with natural compounds. Semin Oncol. 2010; 37: 258-81.

Harborne JB, Williams CA. Advances in flavonoid research since 1992. Phytochemistry 2000; 55: 481-504.

Hartwell LH, Kastan MB. Cell cycle control and cancer. Science 1994; 266: 1821-28.

Hartwell LH, Weinert TA. Checkpoints: controls that ensure the order of cell cycle events. Science 1989; 246: 629-34.

Hunt TR, Schwappach JR, Anderson HC. Healing of a segmental defect in the rat femur with use of an extract from a cultured human osteosarcoma cell-line (SaOS-2). A preliminary report. J Bone Joint Surg Am. 1996; 78: 41-48.

Ishii K, Tanaka S, Kagami K, Henmi K, Toyoda $\mathrm{H}$, 
Kaise T, Hirano T. Effects of naturally occurring polymethyoxyflavonoids on cell growth, p-glycoprotein function, cell cycle, and apoptosis of daunorubicin-resistant $\mathrm{T}$ lymphoblastoid leukemia cells. Cancer Invest. 2010; 28: 22029.

Kim H, Seo E-M, Sharma AR, Ganbold B, Park J,Sharma G, Kang YH, Song DK, Lee SS, Nam JS. Regulation of Wnt signaling activity for growth suppression induced by quercetin in 4T1 murine mammary cancer cells. Int J Oncol. 2013; 43: 1319-25.

Kroemer G, Galluzzi L, Brenner C. Mitochondrial membrane permeabilization in cell death. Physiol Rev. 2007; 87: 99-163.

Lai CS, Li S, Chai CY, Lo CY, Dushenkov S, Ho CT, Pan $\mathrm{MH}$, Wang YJ. Anti-inflammatory and antitumor promotional effects of a novel urinary metabolite, 3',4'-didemethyl nobiletin, derived from nobiletin. Carcinogenesis 2008; 29: 2415-24.

Lai E, Teodoro T, Volchuk A. Endoplasmic reticulum stress: signaling the unfolded protein response. Physiology (Bethesda) 2007; 22: 193-201.

Legrand C, Bour JM, Jacob C, Capiaumont J, Martial A, Marc A, Wudtke M, Kretzmer G, Demangel C, Duval D, Hache J. Lactate dehydrogenase (LDH) activity of the cultured eukaryotic cells as marker of the number of dead cells in the medium. J Biotechnol. 1992; 25: 231-43.

Li RW, Theriault AG, Au K, Douglas TD, Casaschi A, Kurowska EM, Mukherjee R. Citrus polymethoxylated flavones improve lipid and glucose homeostasis and modulate adipocytokines in fructose-induced insulin resistant hamsters. Life Sci. 2006; 79: 365-73.

Luo G, Guan X, Zhou L. Apoptotic effect of citrus fruit extract nobiletin on lung cancer cell line A549 in vitro and in vivo. Cancer Biol Ther. 2008; 7: 966-73.

Luo G, Zeng Y, Zhu L, Zhang YX, Zhou LM. Inhibition effect and its mechanism of Nobiletin on proliferation of lung cancer cells. Sichuan Da Xue Xue Bao Yi Xue Ban. 2009; 40: 449-53.

Ma X, Jin S, Zhang Y, Wan L, Zhao Y, Zhao L. Inhibitory effects of Nobiletin on hepatocellular carcinoma in vitro and in vivo. Phytother Res. 2014; 28: 560-67.

Murakami A, Nakamura Y, Torikai K, Tanaka T, Koshiba T, Koshimizu K, Kuwahara S, Takahashi Y, Ogawa K, Yano M, Tokuda H, Nishino H,Mimaki Y, Sashida Y, Kitanaka
S, Ohigashi H. Inhibitory effect of citrus nobiletin on phorbol ester-induced skin inflammation, oxidative stress, and tumor promotion in mice. Cancer Res. 2000; 60: 5059-66.

Murakami A, Shigemori T, Ohigashi H. Zingiberaceous and Citrus Constituents, 1'-cetoxychavicol Acetate, Zerumbone, Auraptene, and Nobiletin, Suppress LipopolysaccharideInduced Cyclooxygenase-2 Expression in RAW264.7 Murine Macrophages through Different Modes of Action. J Nutr. 2005; 135: 2987S-92S.

Nakajima A, Yamakuni T, Haraguchi M, Omae N, Song SY, Kato C, Nakagawasai O, Tadano T, Yokosuka A, Mimaki Y, Sashida Y, Ohizumi Y. Nobiletin, a citrus flavonoid that improves memory impairment, rescues bulbectomyinduced cholinergic neurodegeneration in mice. J Pharmacol Sci. 2007; 105: 122-26.

Neuhouser ML. Dietary flavonoids and cancer risk: Evidence from human population studies. Nutr Cancer. 2004; 50: 1-7.

Newman DJ, Cragg GM. Natural products as sources of new drugs over the last 25 years. J Nat Prod. 2007; 70: 461-77.

Park HH. Structural features of caspase-activating complexes. Int J Mol Sci. 2012; 13: 4807-18.

Sato T, Koike L, Miyata Y, Hirata M, Mimaki Y, Sashida $\mathrm{Y}$, Yano M, Ito A. Inhibition of activator protein-1 binding activity and phosphatidylinositol 3-hinase pathway by nobiletin, a polymethoxy flavonoid, results in augmentation of tissue inhibitor of metalloproteinases- 1 production and suppression of production of matrix metalloproteinases-1 and -9 in human fibrosarcoma HT-1080 cells. Cancer Res. 2002; 62: 1025-29.

Shi MD, Liao YC, Shih YW, Tsai LY. Nobiletin attenuates metastasis via both ERK and PI3K/Akt pathways in HGFtreated liver cancer HepG2 cells. Phytomedicine 2013; 20: 743-52.

Tang M, Ogawa K, Asamoto M, Hokaiwado N, Seeni A, Suzuki S, Takahashi S, Tanaka T, Ichikawa K, Shirai T. Protective effects of citrus nobiletin and auraptene in transgenic rats developing adenocarcinoma of the prostate (TRAP) and human prostate carcinoma cells. Cancer Sci. 2007; 98: 471-77.

Wu JJ, Cui Y, Yang YS, Jung SC, Hyun JW, Maeng YH, Park DB, Lee SR, Kim SJ, Eun SY. Mild mitochondrial depolarization is involved in a neuroprotective mechanism of Citrus sunki peel extract. Phytother Res. 2013; 27: 564-71. 